\title{
Innovación de procesos: estudio práctico de Aguas de Cartagena S.A. E.S.P. "ACUACAR"
}

\author{
Processes innovation: practice study related to Aguas de \\ Cartagena S.A. E.S.P. "ACUACAR"
}

\author{
Javier Alfonso Mendoza-Betin \\ Aguas de Cartagena S.A., E.S.P. Cartagena, Colombia.
}

\begin{abstract}
Resumen
Objetivo: determinar las consecuencias de la innovación de los procesos y la tecnología en la productividad corporativa, a partir de las propuestas de soluciones de mejora para eliminar o mitigar las pérdidas que presentan los procesos de Aguas de Cartagena S.A. E.S.P. Método: estudio desde un enfoque cuantitativo sustentado en el paradigma positivista. Se aplicó la metodología Lean Production y se utilizó diferentes técnicas de estadística descriptiva para contrastar la hipótesis: la innovación de los procesos y la tecnología redunda en la productividad empresarial. El diseño exploratorio y longitudinal ad hoc estuvo soportado en el ámbito de la gestión de procesos, utilizando como instrumentos auxiliares la revisión documental, la observación, la medición de tiempos y el análisis factorial de la varianza. Resultados: Se encontró que solamente las innovaciones en procesos ejercen influencia determinante en la productividad; no así las innovaciones tecnológicas, cuya incidencia en la optimización de los procesos resultó ser muy limitada. Discusión: a la luz de los resultados obtenidos se infiere que la ruta precisa para la mejora continua no está viabilizada por la incorporación de atributos tecnológicos en los procesos, sino por el poder de la cultura organizacional, la solidez del liderazgo directivo y la gestión de la innovación y el conocimiento. Conclusión: se corrobora que la innovación en los procesos ejerce influencia positiva en la mejora productiva y se descarta que las innovaciones tecnológicas sean un factor determinante en la productividad empresarial.
\end{abstract}

Palabras clave: innovación de procesos, tecnología, productividad.

Clasificacion JEL: C12, D21, M10, O32

\section{Abstract}

Objective: This paper aims to determine the consequences of processes innovation and technology in corporate productivity based on management improvements solutions in order to eliminate the losses shown by Aguas de Cartagena S.A. E.S.P. processes. Method: A quantitative approach based on the positivist paradigm is conducted. In order to test the hypothesis: the innovation of processes and technology results in business productivity, the Lean Production methodology and different descriptive statistics techniques was applied. The exploratory and longitudinal ad hoc design in the field of process management, using documentary review, observation, time measurement and factor analysis of variance as auxiliary instruments was suggested. Results: only innovations in processes exert a decisive influence on productivity; but not technological innovations, whose impact on process optimization proved very limited was found. Discussion: It is inferred that the precise route for continuous improvement is not feasible by the incorporation of technological attributes in the processes, but by the power of organizational culture, the solidity of managerial leadership and the management of innovation and knowledge. Conclusions: The argument that innovation in processes exerts a positive influence on productive improvement. Finally, it is ruled out that technological innovations are a determining factor in business productivity.

Key words: process innovation, technology, productivity.

Recibido: $15-06-19$

JEL Classification: C12, D21, M10, 032

OPEN 


\section{Introducción}

La pertinencia y novedad del presente estudio gravita alrededor de exponer con base en una investigación longitudinal de corte positiva la relación que tienen las variables: innovación de procesos, tecnología y productividad empresarial, en una de las compañías de servicio públicos de la ciudad de Cartagena, en donde según la literatura de la última década esta clase de exámenes y exposición de derivaciones en esta parte del país es bastante escasa. Teniendo en cuenta esto, se generó la necesidad de desarrollar un examen numérico con el fin de analizar los hallazgos a la teoría de la innovación de procesos, partiendo de una empresa mediana que cuenta con 533 colaboradores. De esta forma, se deduce que es conveniente estudiar, con base en pruebas fácticas, el fenómeno aludido. De allí que emerge la siguiente hipótesis: La innovación de los procesos y la tecnología redunda en la productividad empresarial.

A través del método Lean Production (Por su nombre en inglés), la revisión documental, la observación y el análisis factorial de la varianza, determinó la conexidad de las tres últimas variables que se enseñan en la Figura 1 , con lo que se planeó observar y perfeccionar su dependencia, pero a su vez, se demostró teóricamente que la innovación de procesos, la tecnología y la productividad, se encuentran estrechamente asociadas a constructos complementarios: gestión del conocimiento.

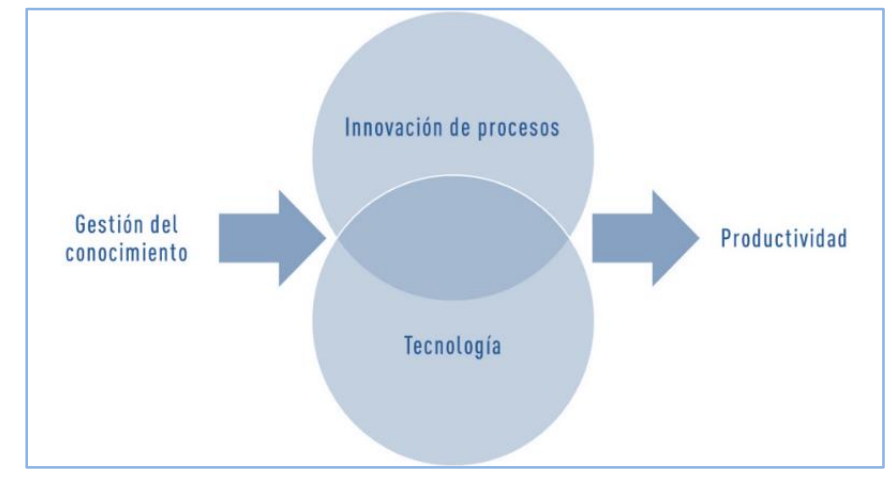

Figura 1. Relación de las variables del estudio

Fuente: Adaptado de Corsino, Mariani y Torrisi (2019), Mendoza-Betin (2018) y Steensma, Chari y Heidl (2015).

\section{Fundamentación teórica}

La mejora continua ha permitido tener rutinas amparadas en políticas de la dirección organizacional, apoyo de teorías del campo de la estrategia auspiciadas en componentes antropomorfos y equipos, de recursos humanos, financieros y materiales, que han posibilitado la gestión y la productividad, bajo el ámbito de innovación y optimización de los procesos. Sin embargo, antes de conocer el trabajo practico, es necesario realizar una aproximación teórica de los paradigmas que permitieron el contraste de los constructos latentes, de tal forma que se pueda demostrar inicial y teóricamente la subordinación de las alterables de la figura anterior (gestión del conocimiento, innovación de procesos, tecnología y productividad), para al final definir y mencionar aspectos importantes de la noción de proceso, de modo que pueda ser detallado, analizado y mejorado en otras circunstancias de interés corporativo. 


\section{Gestión del conocimiento.}

Autores como Wadhwa y Kotha (2006) afirman que la misión del saber se basa en la participación de los inversionistas, las juntas directivas y la alta gerencia en decisiones de inversión, la que es directamente proporcional a la adquisición y explotación de la verdad, conclusión amparada en un escrutinio positivo realizado en empresas de capital de riesgo corporativo en los Estados Unidos. Jensen, Johnson, Lorenz y Lundvall (2007), por su parte, realizaron un examen numérico en el cual dedujeron que la innovación, el uso de la ciencia y la tecnología están supeditada a la producción y el uso de conocimientos científicos y técnicos codificados, lo que requiere de un sistema organizacional competente y óptimo para exteriorizar el discernimiento tácito de los colaboradores.

Asimismo, Wensing, Bosch y Grol (2010) exponen que la exteriorización del conocimiento brota de las interacciones de los colaboradores con sus entornos externos e internos, las cuales necesariamente están ligados a los objetivos de los proyectos establecidos, los que además deben monitorearse por medio de indicadores, previa atenuación o eliminación de las barreras de la administración del saber en las firmas de salud (la animadversión al riesgo, la intolerancia a los errores y la práctica de controlar el cumplimiento del horario laboral).

De manera similar, al menos en las relaciones exteriores de los empleados con sus entornos, Laursen y Salter (2014) argumentan que la misión del conocimiento presenta una relación profunda entre la amplitud de su búsqueda externa y la colaboración grupal y formal para la innovación, y las estrategias de su apropiabilidad.

Leiponen y Helfat (2010) coinciden con Chatterji y Fabrizio (2014) en que el liderazgo y dirección de la deliberación y el discernimiento corporativo implica un sistema serio y a la vez informal pero favorable para su adquisición, asimilación, transformación, demostración y aplicación práctica en todos los eslabones productivos de las organizaciones y sus sectores competitivos (la innovación de procesos, productos, mercados, desarrollos tecnológicos, automatización y robótica). Tanto que Chappin y Ligtvoet (2014); y Shu-Mei y Pei-Shan (2014) con una clara orientación oriental sobre la concepción occidental del conocimiento, aseguran que su gestión se halla desde los sistemas básicos integrados de gestión (calidad, medio ambiental o salud y seguridad en el trabajo) hasta en los métodos y procedimientos más robustos y complejos, representados en base, minería y ciencias de los datos, capaces de mejorar la toma de decisiones estratégicas y tácticas en las firmas.

De la misma forma, autores como Corsino, Mariani y Torrisi (2019); Garzón (2018); Permana, Laksmana y Ellitan (2017); y Wadhwa, Bodas, y Sarkar (2017), que avalan la dirección de la verdad y sin eufemismos, discuten que se trata del activo más importante de la compañía. Para ellos, la administración del conocimiento, brota de una cómoda de experticias, sabidurías, documentos, pericias, rutina de valor, triangulada en los vértices del liderazgo, la cultura y la estructura (formal e informal) que en su interior se encuentra la gente. Además, añaden que el conocimiento emerge de la propia vida de la organización, pero que requiere de la búsqueda y comparación externa y persistente de los procedimientos para absorberlo, reflexionarlo, criticarlo, mejorarlo y emplearlo.

\section{Innovación de procesos y tecnología.}

En relación a la innovación de procesos y la tecnología, autores como Barlow, Bayer y Curry (2006), tratan la combinación de la complejidad en los servicios de medicina local y el contexto político en evolución, así como también 
los factores que influyen tanto en la planificación como en la implementación de una innovación disruptiva, lo que requiere de la integración dinámica de la complejidad de los proyectos, los requisitos de integración (empresa y entorno) y el apoyo transformacional organizativo. Bajo esta guía, se sustenta la rutina de estimulación y el apoyo de ideas de agregación de valor que propicien la mejora continua en su contexto de interacciones. Para estos autores es sustancial destacar la gestión de la innovación dentro de un marco analítico que permita el examen simultáneo y holístico del entorno, la innovación disruptiva de productos, procesos y de mercado, los facilitadores de cambio y el conocimiento, las interacciones entre ellos a lo largo del tiempo, lo que conducido por la alta gerencia, soportada en liderazgo, estrategia y cultura organizacional podrá convertir las ideas en acciones diferentes y aportantes de valor, permitiendo a la empresa competir sustentablemente.

En este sentido, es necesario apuntar que la innovación se torna dinámica cuando los procesos organizacionales cambian y se transforman. Sin lugar a ambigüedades, Damanpour, Walker y Avellaneda (2009), destacan que la destreza de la organización para componer, constituir y reajustar la gestión de la innovación en orden de atender las transformaciones en el entorno, los propios cambios corporativos, productos, nuevos mercados y de procesos, se manifiesta en la conciencia colectiva innovadora, en otros términos, se armoniza de procesos sociales (interacción y reflexión), de la cooperación, examen y evaluación de ideas productivas (íntima y externamente) que cambien el negocio, pero siempre dirigiendo esfuerzo hacia un tipo de innovación.

En este orden de ideas, Atal y Bar (2010) defienden la gestión de la innovación. Para ellos, los innovadores tienen el deber de divulgar cualquier resultado innovador obtenido, y de igual forma, dar a conocer el método que erigieron para enseñar dichas conclusiones. No obstante, señalan que la invención requiere de incentivos económicos a fin de buscar permanentemente las técnicas de creatividad y disposición de ideas periódicas, la búsqueda y la oportunidad de obtenerlas; además advierten, que lo señalado radica en los colaboradores, los que deben trabajar en un sistema eficazmente colaborativo.

Dejando a salvo el relieve que posee la gestión grupal de ideas provechosas para el análisis de la innovación estratégica institucional, es oportuno vincular el constructo de innovación abierta de Dahlander y Gann (2010), lo que es una clara emulación al principio de destrucción creativa propuesto por Schumpeter, lo que en última, significa reconfigurar el pensamiento y accionar estratégico de forma recurrente. Particularmente hacen referencia a la improvisación responsable como elemento de orden, aunque se razone tautológico. En la misma vertiente, el paradigma de Baldwin y Von Hippel (2011) sostiene que la innovación por individuo al igual que la de colaboración abierta compite cada vez más, y pueden desplazar, la de productores en muchas partes de la cadena productiva de la economía.

Del mismo modo, Lampe (2012), discute y recomienda que la innovación aborda actividades iterativas conducentes al perfeccionamiento y ajuste perenne de los procesos de funcionamiento estratégico y táctico debido a la transformación de ideas en productos, procesos, desarrollos tecnológicos o mercados diferentes. Sin embargo, asegura que convendrán otras operaciones de orden valioso que le provean el factor sorpresa, auspiciado en la cultura innovadora disruptiva, en otras palabras, aprovechar los elementos con los que cuenta la empresa para beneficio propio, lo que hace alusión al trabajo Dalvano e Hidalgo (2012) y el método de la empresa SIT Innovation (2019), quienes declaran que son hábitos industriales que apremian reducir las desidias competitivas, mediante la promoción sistemática y sistémica de rutinas diferenciales propias. 
Por otro lado, la corriente que defienden Vasileiou, Barnett y Young (2013) sostiene que la innovación no escala por condición y subordinación sine qua non con la tecnología. Los resultados de su trabajo de paradigma mixto esgrimen que los innovadores articulan conceptos prácticos de los enfoques principales de la innovación (productos, mercado, desarrollos tecnológicos y procesos) en relación con ensayos clínicos, lecciones aprendidas, resolución de problemas y ciclos de mejora continua. Bajo la implementación de estos métodos, lo cual se basó en la estrategia, cultura y liderazgo innovador, con lo que pudieron demostrar que bajo pruebas amparadas en trabajo de equipo y corolarios pragmáticos enmarcados todos en ciclos de mejora, obtuvieron "mejores" derivaciones transformadoras. En conclusión, determinaron que el desarrollo de mecanismos de apoyo y la evaluación de la innovación logró optimizar rutinariamente la eficiencia de la organización, lo que no necesariamente tiene ahínco en la tecnología.

Autores como Arora, Cohen y Walsh (2016) afirman que toda firma opera bajo un régimen dual de innovación, por una parte, en unión definitiva con el medio ambiente, y por otra, desde la corporativa. En tal sentido, sus datos y conclusiones sugieren que el progreso y la mercantilización de la innovación recae en la mayor división del trabajo entre inventores e innovadores, en la consecución de oportunidades o manchas grises (no atendidas) en la cadena de agregación de valor, específicamente en sus fuentes externas, pero que se ha descuidado lamentablemente la comprensión y aprovechamiento de los componentes internos que condicionan, así mismo, los determinantes de la velocidad y dirección de la actividad innovadora, lo que escala en la competitividad organizacional diferenciada y productiva.

Desde otro punto de vista, Coffano y Tarasconi (2014), armonizando con Bettis, Helfat y Shaver (2016), reconocen que la innovación es un proceso de alto rendimiento para producir diferencias sustanciales en el campo estratégico y operativo, asienten que los ingredientes que motivan dicho desempeño superior, de ahí su razonamiento sobre efectuar exámenes de este corte, a merced de analizar dicha fenomenología, se basa en un gobierno que viabilice el suministro de este activo estratégico empresarial mediante un método lógico, dinámico y de respuesta permanente.

La posición de Robayo (2016) es que el ejercicio empresarial está claramente ligado al fenómeno, es decir, el constructo operacionalizado es estimado como un proceso y no un resultado final. De hecho, insisten que la conquista de la gerencia de la innovación incluye una cultura relacionada con la proposición de ideas y su gestión por medio de un sistema formal de incentivos, que facilite determinar los factores críticos de competitividad que especifican dicho proceso, de tal suerte que se potencie su desarrollo y primeramente su establecimiento iterativo. De esto, se deduce que la cultura organizacional deberá gestionar el conocimiento implícito de los colaboradores, con lo que se esperan cambios positivos en la competitividad y sostenibilidad.

El discurso de Álvarez, Serrano y Bravo (2017) gira en torno a la pertinencia de investigar sobre métodos que faciliten la implementación de la innovación; sin embargo, a su vez, coligen en la importancia de intercambio de los niveles de adiestramiento y formación, los saberes alcanzados y las circunstancias adaptativas de discernimientos que conduzcan a la gestión del conocimiento, y bajo esta vía a la innovación sostenible. Una práctica recurrente para este objetivo es la comparación con empresas del sector. 
Aquí corresponde detenerse un instante para resaltar que, los conceptos vistos hasta esta parte y analizados en el primer escenario conceptual se relacionan entre sí y con el de las capacidades dinámicas de innovación, la que incluye entre sus líneas de acción la innovación de procesos. Empero, por otra parte, es claro que la tecnología se convierte en una ramificación de la innovación, a ciencia cierta, ésta hace parte y es la consecuencia de la innovación, la creatividad y la invención. En síntesis, la revisión de la literatura anterior demuestra la relación hipotética de la dirección del saber, la innovación de procesos y la tecnología.

\section{Productividad.}

Vista la relación teórica de las variables anteriormente abordadas, es el turno ahora de la productividad. Para colegir su definición, se principiará con la proposición de la Norma Internacional ISO 9001 (2000; 2015) sobre procesos, cuyo paradigma auspiciado en la inclinación rutinaria es uno de los compendios de la gestión de la calidad, la que la define como un conjunto de actividades recíprocamente afines o que interactúan, transmutando elementos de entrada en consecuencias, cimentado en un gobierno amparado en el ciclo de Deming (1989) o PHVA, que alcanza y completa los escenarios de estudio y monitoreo continuo del entorno de la compañía, entre otros; la dirección y pensamiento estratégico, la planeación de la calidad, los recursos físicos y humanos, y la gerencia del conocimiento.

Bajo el mismo discurso, se agrega que los procesos instituyen para un número importante de organizaciones su estructura estratégica, dada la flexibilidad que dispone esta gestión para acomodarse a las habituales transformaciones en el entorno y el mercado. De hecho, concentra su interés en las rutinas aportantes de ajuste, cambio y valor porque representa la palanca más poderosa para actuar sobre los resultados de manera efectiva y sostenible.

Existen numerosos métodos para actuar y mejorar los procesos, entre otros; el procedimiento sistemático de mejora para cambios graduales, la reingeniería y el Lean Production. Este último método, es el que se incorporó al presente ejercicio práctico.

\section{Método Lean Production.}

La Metodología Lean Production (por su nombre en inglés), se define como "Pérdidas" lo que discurren como las acciones que no añaden valor, lo que es lo mismo a acotar a generar costos en los procesos.

Desde otro punto de vista, este término pretende resarcir estos defectos por medio del mejoramiento, delimitándose a eliminación de las pérdidas por medio de la eficiencia (progreso perpetuo y desarrollos tecnológicos).

De la misma forma, se consideran pérdidas, todo lo que sea una constricción, limitación o retrasos, es decir, lo que no agrega valor a los productos o procesos, de conformidad a lo expuesto por Alarcón y Campero (1999, p. 322), y que al eliminarlos o mitigarlos se incrementa la productividad (Alarcón, 1997, p. 497). Es así como Caro y González (2015), agregan que, el proceso se delimita muy eficiente cuando produce una altísima calidad, y en consecuencia, existen pocos desperdicios o porque su ciclo de respuesta es muy corto. En sinopsis, la productividad se delimitará operativamente con la disminución de las mermas o menguas en los procesos, entre otros, como los que se muestran en la Figura 2. 


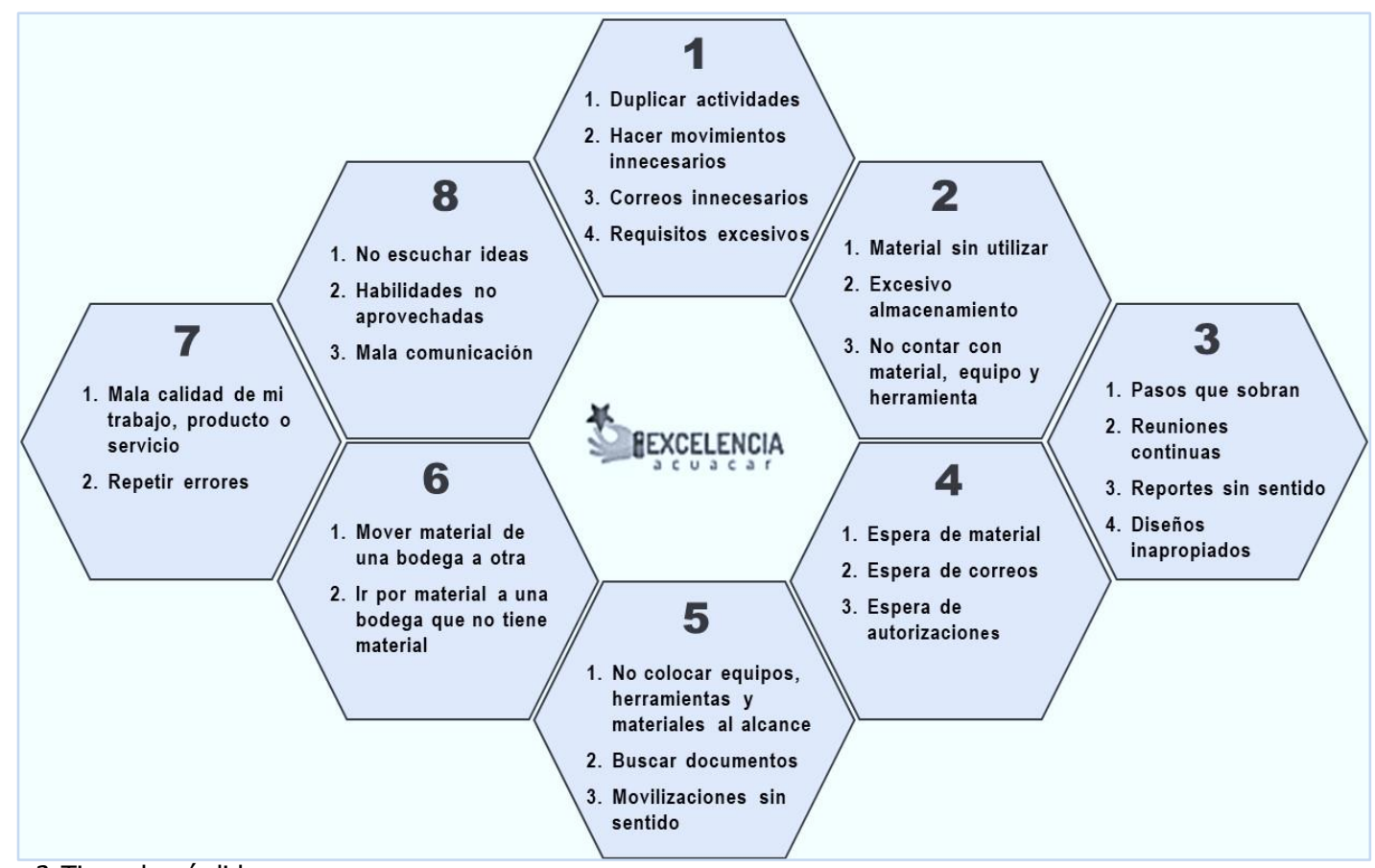

Figura 2. Tipos de pérdidas

Fuente: Tomado de Acuacar (2019) basado en Botero y Álvarez (2003).

\section{Método}

En orden de lograr el propósito de este trabajo empírico, se utilizó un estudio exploratorio de corte longitudinal por espacio de 2 años, basado en el método Lean Production y en el paradigma de procesos. Asimismo, se utilizaron como instrumentos complementarios técnicas estadísticas.

El método abordado consistió en cuatro etapas con propósitos marcados. Cada ciclo constituyó una seria de actividades, las cuales fueron formuladas de conformidad a su ocurrencia, pero también arrojaron datos los cuales se debieron evaluar estadísticamente.

\section{Ełapa 1: Comprensión de los procesos e identificación de problemas.}

(1) Identificación de los procesos de la empresa: esta actividad tuvo como finalidad establecer y especificar los procesos con cada una de sus interrelaciones.

(2) Identificación del conflicto: tuvo como intención documentar y analizar el estado actual de los procesos detectando constricciones o retrasos en su desempeño. Para esto, se formaron unas subfases:

a. Diagnóstico: se detectaron los problemas que afectaban la misión de la organización.

b. Identificación de los procesos, subprocesos con mayores incidencias en los conflictos determinados. 


\section{Etapa 2: Análisis y escrutinio de los subprocesos identificados.}

Este período tuvo por propósito estudiar detalladamente los procesos o subprocesos, descomponiéndolos en unidades de análisis inferiores (actividades y tareas). De esto, se determinaron los retrasos que des-optimizaban los procesos para con base en estos se pudieran proponer iniciativas (palancas de mejora) para su eliminación o mitigación. Esta etapa abarcó las acciones mencionadas a continuación:

a. Descripción del subproceso en el cual se aforan los retrasos enmarcados al interior de los siguientes tipos de desperdicios, incluyendo sus respectivos tiempos para cada constricción.

b. Experimento. Recolección y acopio de datos.

c.Realización de aforos.

d. Análisis, presentación y socialización de las consecuencias.

\section{Ełapa 3: Mejoramiento.}

(1) Discusión y aforos con el personal administrativo y operativo de las limitaciones que causaron los retrasos, presentando iniciativas-palancas de mejora-para eliminarlas o mitigarlas.

\section{Etapa 4: Definición de variables.}

(1) Revisión de los elementos obtenidos en las etapas anteriores, con la intención de definir las variables conceptuales y operacionales.

La Tabla 1 sintetiza las técnicas y herramientas estadísticas usadas en cada etapa.

Tabla 1.

Técnicas implementadas en las etapas del trabajo

\begin{tabular}{|c|c|c|}
\hline $\begin{array}{r}\text { Etapa } \\
\end{array}$ & $\begin{array}{c}\text { Actividades } \\
\end{array}$ & Técnicas implementadas \\
\hline \multirow[t]{2}{*}{$\begin{array}{l}\text { 1. Comprensión de los procesos e } \\
\text { identificación de problemas }\end{array}$} & $\begin{array}{l}\text { Identificación de los procesos de la } \\
\text { empresa }\end{array}$ & Revisión documental \\
\hline & Identificación del conflicto. & $\begin{array}{l}\text { Revisión documental, muestreo, } \\
\text { estadística descriptiva, reuniones, } \\
\text { observación }\end{array}$ \\
\hline \multirow[t]{4}{*}{$\begin{array}{l}\text { 2. Análisis y escrutinio de los } \\
\text { subprocesos identificados }\end{array}$} & Descripción de los subprocesos & $\begin{array}{l}\text { Revisión documental, observación, } \\
\text { diagramas de flujo }\end{array}$ \\
\hline & $\begin{array}{l}\text { Experimento. Recolección y acopio de } \\
\text { datos. }\end{array}$ & $\begin{array}{l}\text { Revisión documental, observación, } \\
\text { medición de tiempo }\end{array}$ \\
\hline & Realización de aforos. & $\begin{array}{l}\text { Medición de tiempos, estadística } \\
\text { descriptiva }\end{array}$ \\
\hline & $\begin{array}{l}\text { Análisis, presentación y socialización de los } \\
\text { resultados. }\end{array}$ & Estadística descriptiva \\
\hline 3. Mejoramiento & $\begin{array}{l}\text { Discusión y aforos con el personal } \\
\text { administrativo y operativo de las } \\
\text { limitaciones que causan los retrasos }\end{array}$ & $\begin{array}{l}\text { Medición de tiempo, estadística } \\
\text { descriptiva y observación }\end{array}$ \\
\hline
\end{tabular}




\section{Definición de variables \\ Revisión de los elementos obtenidos en las etapas anteriores, con el propósito de definir las variables conceptuales y operacionales \\ Análisis de los resultados, indicadores, definición de variables operativas y las unidades de medición, de tal manera que se pueda demostrar la hipótesis.}

Nota. Fuente: Tomado de la metodología Lean Production de Botero y Álvarez (2003) y de Acuacar (2019).

Una vez se avanzó en la definición de las variables conceptuales y operacionales se acopiaron los datos de las transformables independientes y dependientes, los cuales resultaron de la aplicación de instrumentos de recogida de información sometido a un doble proceso de validación: 1) mediante juicio de 3 expertos y 2) a través de una prueba piloto en la que participó una muestra intencional de 10 directivos de ACUACAR. Luego de la prueba piloto, se administró dicho mecanismo a los colaboradores participantes de las sesiones semanales del Plan de Excelencia como se acota más adelante.

\section{Variables Independientes.}

\section{Innovación de procesos.}

La innovación de procesos se evaluó utilizando una escala ad hoc establecida por el Plan de Excelencia de ACUACAR, y ajustada con fundamento en las consultas teóricas, la cual fue creada bajo la siguiente dimensión hipotética, coincidiendo con Robayo (2016): Iniciativas de mejora entregadas por los colaboradores de ACUACAR.

\section{Tecnología.}

Por su parte, la Tecnología se midió escrutando una escala establecida también por el Plan de Excelencia de ACUACAR, y mejorada con sustento en las extensiones teóricas Steensma, Chari y Heidl (2015). Esta métrica contiene la dimensión y factor denominado: Iniciativas entregadas por los colaboradores que se materializaron en desarrollos tecnológicos.

\section{Variables Dependientes.}

\section{Productividad.}

El rendimiento se mide en conexidad a la eficiencia de los procesos, eliminado las pérdidas o retrasos, amparado en el método Lean Production, el que se encuentra respaldado por el ejercicio de Botero y Álvarez (2003).

Con base en la información de la última etapa descrita, se acometió determinar la conexidad de las tres últimas variables mencionadas, y aún mejor, calcular y evaluar los efectos de las alterables (innovación de procesos y tecnología) sobre la productividad, auspiciado en un esquema de relación causal multivariada, específicamente el análisis factorial de varianza (ANOVA), el que se dedujo al tener acceso a los datos del fenómeno. En términos generales, de conformidad al escrutinio literario, la relación y efecto entre las variables ha sido estudiado internacionalmente, mas no así en el escenario local de una empresa de Cartagena. Teniendo en cuenta el vacío de discernimiento, se propone la siguiente hipótesis: 
Hipótesis H1: A mayor innovación de procesos y tecnología mejora la productividad corporativa.

La muestra incluyó 533 unidades de análisis, todos colaboradores de la empresa, quienes participan en un plan de mejoramiento de los procesos denominado "Plan de Excelencia", el que inicio a partir de junio de 2017 y cuya evaluación de corte empírico se hará al exponerlo en una revista internacional indexada.

\section{Observaciones}

Al margen de la revisión documental, aforos de tiempos, análisis de resultados, y las observaciones, permitieron acceder a la información directa del estudio, en el cual tienen lugar la facilitación del conocimiento, el trabajo grupal y las decisiones de los directivos, mandos medios y operarios de la corporación y, categóricamente, suministraron realidades sobre el comportamiento de sus integrantes, y al final fortalecerá las conclusiones de la investigación. En lo relativo a la comprensión de la situación analizada, se efectuaron recorridos en donde semanalmente se realizó el Plan de Excelencia.

\section{Resultados}

De acuerdo con el diseño metodológico empleado se logró encontrar que el análisis factorial de la varianza era la técnica metodológica más conveniente, en razón de que cumple con los determinadores para evaluar la derivación de dos o más inconstantes autónomas (innovación de procesos y tecnología) sobre una variable dependiente (productividad), puesto que además posibilita estimar y ajustar las relaciones de las transformables observables (Cruz y Koch, 2015; Hollon, 2006). Sin embargo, es de anotar que se utilizó un proceso longitudinal de cuatro pasos para recoger, estudiar y ajustar los datos contrastados, que incluyó en la primera parte la comprensión de los procesos corporativos. En la segunda posición, se analizaron y escrutaron los subprocesos identificados, en tercer lugar, se midió el mejoramiento de los procesos y en el cierre, se operacionalizaron las variables.

Los resultados de las etapas se presentan a continuación:

Para comprender mejor los procesos se dividió el Plan de Excelencia de Acuacar en tres hitos: subprocesos, actividades y tareas, en estas últimas se aforaron su duración y las perdidas (retrasos) con lo que se determinó la línea base y unidades de medición de la productividad. Al obtener estos resultados, se estableció que la mejor forma para eliminar o mitigar las perdidas está relacionada con la participación de los colaboradores de la compañía por medio de ideas, las que se proferían, esgrimían y evaluaban semanalmente en centros y repositorios de conocimiento físicos, grupales y digitales (Haciendo alusión al "BA" enunciado por Nonaka y Noburo (1998), quienes sostienen que son sitios para crear discernimiento organizacional), de conformidad a la Figura 3. La innovación de procesos está subordinada al aforo de los colaboradores de identificar oportunidades de mejora (retrasos), mediante una apropiada gestión de iniciativas (Acuacar, 2019). 


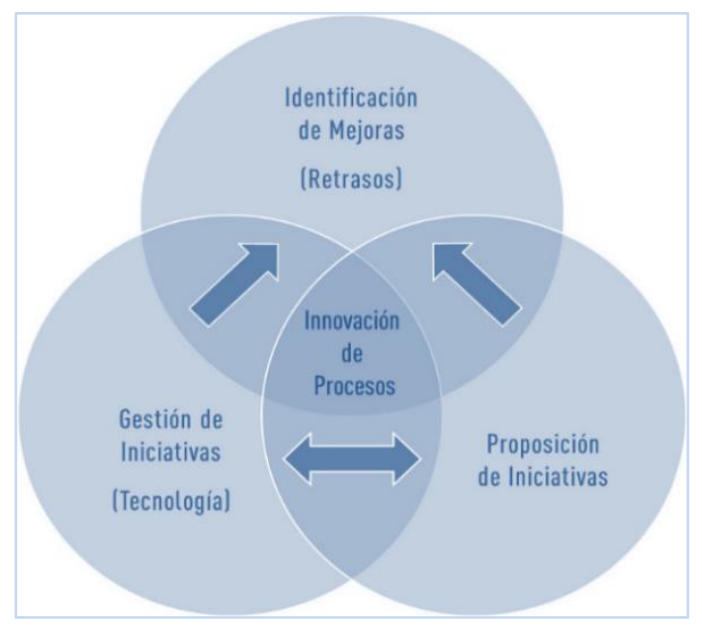

Figura 3. Ciclo de mejoramiento

Fuente: Acuacar (2019) basado en Permana, Laksmana y Ellitan (2017); Wadhwa, Bodas, y Sarkar (2017).

Bajo este sistema mencionado, se atendieron, analizaron y evaluaron un número importante de ideas (Acuacar las denomina iniciativas o palancas de mejora) en el periodo 2017 y 2019, entre las que se encuentran varios tipos: por un lado, dotación de vehículos, herramientas y equipos, capacitación al personal, optimización de proveedores de back y front office, actualización documental de procedimientos e instrucciones de trabajo, y por otro lado, se redireccionaron hacia la automatización de los procesos por medio de desarrollos tecnológicos, lo cual al momento de proveérsele a los empleados y las rutinas, se fue calculando y revisando el efecto en la productividad, todo esto se encuentra consolidado en la Tabla 2, es decir, los datos observables de las variables operacionales.

\section{Tabla 2.}

Acopio de las variables conceptuales y operacionales del trabajo

\begin{tabular}{|c|c|c|c|c|}
\hline Meses & $\begin{array}{l}\text { Productividad - \% } \\
\text { Eficiencia Procesos }\end{array}$ & Iniciativas & $\begin{array}{c}\text { Iniciativas } \\
\text { consideradas como } \\
\text { desarrollos } \\
\text { tecnológicos }\end{array}$ & $\begin{array}{c}\text { Participación } \\
\text { desarrollos } \\
\text { tecnológicos } \\
(\%)\end{array}$ \\
\hline jun-17 & $53 \%$ & & & \\
\hline jul-17 & $52 \%$ & & & \\
\hline ago-17 & $51 \%$ & 50 & 23 & $46 \%$ \\
\hline sep-17 & $53 \%$ & 37 & 14 & $38 \%$ \\
\hline oct-17 & $50 \%$ & 42 & 14 & $33 \%$ \\
\hline nov-17 & $53 \%$ & 83 & 26 & $31 \%$ \\
\hline dic-17 & $52 \%$ & 137 & 52 & $38 \%$ \\
\hline ene-18 & $55 \%$ & 109 & 42 & $39 \%$ \\
\hline feb-18 & $56 \%$ & 107 & 22 & $21 \%$ \\
\hline mar-18 & $56 \%$ & 73 & 8 & $11 \%$ \\
\hline abr-18 & $53 \%$ & 170 & 48 & $28 \%$ \\
\hline may-18 & $63 \%$ & 131 & 29 & $22 \%$ \\
\hline jun-18 & $64 \%$ & 92 & 14 & $15 \%$ \\
\hline jul-18 & $68 \%$ & 77 & 12 & $16 \%$ \\
\hline ago-18 & $70 \%$ & 92 & 13 & $14 \%$ \\
\hline sep-18 & $71 \%$ & 193 & 47 & $24 \%$ \\
\hline oct-18 & $67 \%$ & 256 & 56 & $22 \%$ \\
\hline
\end{tabular}




\begin{tabular}{ccccc}
\hline nov-18 & $67 \%$ & 156 & 33 & $21 \%$ \\
\hline dic-18 & $69 \%$ & 88 & 18 & $20 \%$ \\
\hline ene-19 & $69 \%$ & 159 & 49 & $31 \%$ \\
\hline feb-19 & $72 \%$ & 96 & 26 & $27 \%$ \\
\hline mar-19 & $72 \%$ & 4 & & 0 \\
\hline abr-19 & $73 \%$ & & & \\
\hline may-19 & $74 \%$ & & 546 & $25 \%$ \\
\hline jun-19 & $75 \%$ & 2152 & & \\
\hline Total & & & & \\
\hline
\end{tabular}

Fuente: Acuacar (2019).

No obstante, todo lo anterior y la cantidad importante de retrasos identificados (uno por cada iniciativa), se aplicó una encuesta a una muestra de 10 trabajadores de los 43 que conforman el equipo líder para explorar, analizar y categorizar los principales factores que a su criterio retrasan los procesos. Para cada reactivo se presentaba una escala de valoración del (1) al (5), siendo (1) la declaración de estar totalmente en desacuerdo, mientras que (5) encarna estar totalmente de acuerdo. Los colaboradores se escogieron a través de un muestreo no probabilístico. Del estudio de las consecuencias del instrumento y la aplicación del indicador: alfa de Cronbach (citado por Martin \& Bridgmon, 2012), se concluye que en términos generales:

Con base en los criterios de Huh, Delorme y Reid (2006), los efectos que exhibe la Tabla 3 prueban una buena consistencia de los factores analizados, lo que en otras palabras quiere decir que se consigue un nuevo instrumento de medición de cara a la comunidad científica empresarial, al que solo restaría aplicarle un juicio de expertos.

Tabla 3.

Estadísticos de fiabilidad en los factores que originan los retrasos

\begin{tabular}{ccc}
\hline & \multicolumn{2}{c}{ Estadísticos de Fiabilidad } \\
\hline Alfa de Conbrach & Alfa de Conbrach basada en los elementos tipificados & N de Elementos \\
\hline 0,875 & 0,873 & 10 \\
\hline
\end{tabular}

Fuente: Elaboración propia en SPSS (2019).

Los datos de la Tabla 4 permiten colegir aún mejor que al obtener factores reactivos-total ajustados, para los componentes destacados en color rojo, los que son inferiores a 0.3 , se apartarán del cuestionario, y en consecuencia solo resultan 7 componentes principales como génesis de los retrasos en los procesos de 10 evaluados.

Tabla 4.

Estadísticos Total - Factores que originan los retrasos

\begin{tabular}{|c|c|c|c|c|c|}
\hline $\begin{array}{l}\text { Factores que originan } \\
\text { los retrasos }\end{array}$ & $\begin{array}{c}\text { Media de la } \\
\text { Escala si se } \\
\text { elimina el } \\
\text { elemento }\end{array}$ & $\begin{array}{l}\text { Varianza de la } \\
\text { Escala si se } \\
\text { elimina el } \\
\text { elemento }\end{array}$ & $\begin{array}{c}\text { Correlación } \\
\text { elemento - total } \\
\text { corregida }\end{array}$ & $\begin{array}{l}\text { Correlación } \\
\text { Múltiple al } \\
\text { cuadrado }\end{array}$ & $\begin{array}{c}\text { Alfa de Cronbach } \\
\text { si se elimina el } \\
\text { elemento }\end{array}$ \\
\hline Tecnología & 74,89 & 52,099 & 0,524 & . & 0,869 \\
\hline Proveedores & 74,61 & 52,692 & 0,431 & . & 0,871 \\
\hline $\begin{array}{c}\text { Comunicación con los } \\
\text { usuarios }\end{array}$ & 75,36 & 46,46 & 0,641 & . & 0,863 \\
\hline $\begin{array}{c}\text { Contratos sin acciones } \\
\text { correctivas }\end{array}$ & 75,21 & 46,545 & 0,674 & . & 0,861 \\
\hline
\end{tabular}




\begin{tabular}{ccccc}
\hline $\begin{array}{c}\text { Interventoría no adecuada; } \\
\text { no aplican las acciones } \\
\text { correctivas de los contratos }\end{array}$ & 74,75 & 53,972 & 0,336 & 0,874 \\
\hline Personal no competente & 75,25 & 46,935 & 0,709 & $\cdot$ \\
\hline Rotación de personal & 76 & 49,111 & 0,572 & 0,86 \\
\hline Actos de corrupción & 74,68 & 54,226 & 0,25 & 0,866 \\
\hline $\begin{array}{c}\text { Agilidad de las operaciones, } \\
\text { capacidad de reacción }\end{array}$ & 74,89 & 55,655 & 0,057 & 0,876 \\
\hline Capacidad de reacción & 74,71 & 54,286 & 0,212 & 0,88 \\
\hline
\end{tabular}

Fuente: Elaboración propia en SPSS (2019).

En tal virtud, se determinaron e identificaron las 7 primeras variables observables como problemas a estudiar dentro de otro alcance investigativo, los cuales enseñan los principales retrasos en la ejecución de los procesos, de los que sería importante determinar sus causas y de estas, sus micro retrasos.

- Tecnología. Indican que el atraso tecnológico se encuentra en la capacidad de la organización para analizar la multidimensionalidad de las variables y las extensas bases de datos. Sin embargo, es menester referirse al automatismo con los proveedores de servicios por medio de un canal de comunicaciones digital, competente y pertinente para resolver las constricciones en materia de asignación de trabajo, recibo de trabajo, manejo adecuado de los inventarios, pago a los contratistas y cobro a los usuarios del servicio.

- Proveedores. Es necesario mayor acompañamiento a los proveedores de servicios en materia de escogencia del personal, desarrollo por competencias y gestión integral y eficaz de sus procesos.

- $\quad$ Comunicación con los usuarios.

- Contratos sin acciones correctivas. Adecuación contractual.

- Interventoría no adecuada. Capacitación y formación permanente en la administración contractual.

- Personal no competente, especialmente en atención y trato al usuario (Acuacar y proveedores de servicios). Desarrollo y formación de competencias blandas.

- Rotación de personal operativo de contratistas. Garantizar una única estructura de salarios para los operadores de los contratistas.

La recopilación de datos de la empresa, y en especial los del Plan de Excelencia, relacionados con las variables en los últimos dos años consintió disentir la hipótesis obteniendo que solo la variable, iniciativas generales presenta efectos positivos (sig. 0,018) sobre la productividad. La Tabla 5 que acopia los resultados del contraste factorial de la varianza (ANOVA), confirma el resultado anterior.

\section{Tabla 5.}

Estadístico - Resultado del ANOVA de 3 factores. Pruebas de efectos inter-sujetos

Variable dependiente: Eficiencia de procesos

\begin{tabular}{cccccc}
\hline Origen & $\begin{array}{c}\text { Tipo III de suma } \\
\text { de cuadrados }\end{array}$ & gl & Media cuadrática & F & Sig. \\
\hline Modelo corregido & $0,101^{\text {a }}$ & 5 & 0,02 & 4,518 & 0,007 \\
\hline Intersección & 4,564 & 1 & 4,564 & 1022,594 & \\
\hline
\end{tabular}




\begin{tabular}{cccccc}
\hline Iniciativas generales & 0,044 & 2 & 0,022 & 4,983 & 0,018 \\
\hline Iniciativas tecnológicas & 0,001 & 2 & 0,001 & 0,132 & 0,877 \\
\hline Iniciativas generales * Iniciativas tecnológicas & 0 & 0 & $\cdot$ &. \\
\hline Error & 0,085 & 19 & 0,004 & \\
\hline Total & 9,862 & 25 & & \\
\hline Total corregido & 0,186 & 24 & & \\
\hline
\end{tabular}

Nota. Fuente: Elaboración propia valoradas en SSPS (2019).

a. $\mathrm{R}$ al cuadrado $=, 543(\mathrm{R}$ al cuadrado ajustada $=, 423)$

\section{Discusión y conclusiones}

La investigación longitudinal de corte positivo sobre los efectos de las alterables (innovación de procesos y tecnología) en la productividad arrojó mediante el análisis factorial multivariado que solo la primera enseña derivaciones positivas (sig. 0,018 ) en la productividad concordando parcialmente con la visión hipotética de Corsino et. al., (2019) y Steensma, Chari y Heidl (2015), rechazando en esta oportunidad que la tecnología sea análoga a la variable mencionada al menos en esta fenomenología. Sin perjuicio de este resultado, los hallazgos ad hoc confirman teórica y empíricamente de manera débil y limitada $(0,877)$ que la relación de la tecnología ayuda a la optimización de procesos de Aguas de Cartagena S.A. E.S.P., por lo que se infiere que el Plan de Excelencia deberá exhortar aún más a la presentación de palancas de mejora de este ámbito.

No obstante, los descubrimientos corroboran también la tesis de Coffano y Tarasconi (2014), en la cual se ampara que "cuando se innovan los procesos la producción de la empresa mejora", con lo que se deduce por sustracción de materia o inferencia razonable que el Plan de Excelencia como gestor de conocimiento y medio ambiente benéfico para la generación, tratamiento y evaluación de ideas es la ruta precisa para la mejora continua, dados los integrantes aludidos por dicho autor: ideas clave y productividad. Sin embargo y al margen de lo anterior, se destaca la jerarquía de la cultura y liderazgo de la gerencia general y su equipo directivo, lo que sin lugar a duda viabiliza la coexistencia propicia de este tipo de situaciones, variables, métricas y datos en las organizaciones. Estas manifestaciones tienen el potencial de prodigar implicaciones para la academia, el sector real y proyectar líneas promisorias de investigaciones en empresas grandes.

\section{Implicaciones para el estado del arte y la gestión gerencial}

De cara a la actualización de la literatura, el procedimiento, las técnicas del escrutinio de corte cuantitativo, sumado a los resultados obtenidos, es notable y loable señalar que interesarán como referencia y consulta a la academia y el sector real. El rol sobresaliente que goza la gestión del conocimiento y la innovación al interior de las organizaciones para aumentar la productividad se convertirá en un alto en el camino para la reflexión organizacional, y sobre todo para la implementación de prácticas como las que enseña el Plan de Excelencia de ACUACAR.

Por otra parte, el examen científico requirió que las variables operacionales: iniciativas en general e iniciativas tecnológicas que representan las alterables conceptuales: innovaciones de procesos y la tecnología, respectivamente, se contrastaran con la literatura, resultando viable su operatividad. Una vez revisadas bajo este ámbito, se encontró 
su medición en números enteros (cantidad mensual de cada una), por lo que hubo necesidad de cambiarlas a unidades ordinales y asignarles rangos de atributos. Se computaron en consecuencia en una escala Likert, siendo 0,75 los de valores óptimos, las iniciativas menormente terminadas. De esta forma, se aplicó el ANOVA, lo que posibilitó contrastar la hipótesis cardinal, validando y probando parcialmente el estado del arte expuesto.

Al margen de las constricciones presentadas en el uso de la técnica señalada, este análisis brinda líneas prominentes de investigación a los equipos gerenciales, la ciencia de la administración y al sector real sobre el desarrollo y gestión de las variables acotadas. Los sistemas de mejoramiento como los que representa el Plan de Excelencia posibilitan el liderazgo, cultura organizacional, la gestión de la innovación y conocimiento mediante la formación y desarrollo de habilidades y rutinas de primer orden competitivo, despertando concepciones esenciales, estratégicas y constructos que caracterizan a las corporaciones sostenibles.

\section{Limitaciones}

Una primera constricción es que el ejercicio no incluyo los proveedores de servicios, los que podrían contar con unos 400 colaboradores aproximadamente, incluyendo los órganos de gobierno. En el corto plazo podrían incorporarse a estudios posteriores, las organizaciones de cara a los usuarios del servicio de acueducto y alcantarillado, lo cual implica recursos fuera del alcance de este primer escrutinio.

Adicionalmente el método e instrumentos de medición ad hoc, se aplicaron en un plazo de dos años, en tal virtud, no posibilitó conocer los hallazgos de las unidades de análisis en plazos más extensos, lo cual ayudaría a generalizar la Teoría de la innovación de procesos. Por su parte, no fue posible levantar la información asociada a la variable de gestión del conocimiento, puesto que no hace parte del alcance del Plan de Excelencia. No obstante, se observa el Plan de Excelencia como un muy buen gestor de conocimiento y medio benefactor para la creación, sistema y valoración de ideas para la mejora continua, lo cual es reconocido por todos los empleados de ACUACAR.

Finalmente, aun así, se logra establecer limitaciones en el alcance del escrutinio y la particularidad de la empresa analizada, en próximas avenidas de investigaciones podría extenderse del estudio a los proveedores de servicio, a condición de generalizar los hallazgos.

\section{Futuras líneas de investigación}

Descontado los trabajos teóricos y numéricos realizados, es pertinente mayor investigación coligada a la innovación de procesos, al igual que su conexidad con la gestión del conocimiento, la tecnología y la productividad, mejor aún, para diferentes sectores productivos, micros, mediana y grandes organizaciones, especialmente en el contexto local y en específico en la región caribe colombiana. Finalmente, como parte del presente inciso, es necesaria mayor investigación práctica que valide la relación de las transformables acotadas.

Otras líneas de investigación están basadas en la cultura organizacional, el desarrollo de competencias blandas, la relación de estos escenarios teóricos con las CD dentro del escenario de la estrategia de las MIPYMES colombianas, el análisis de las causas de los factores principales asociados a las pérdidas y la determinación y mitigación o eliminación de las causas acotadas, al igual que micro retrasos, y en consecuencia sobre la productividad y otros factores 
competitivos sostenibles. En conclusión, el método y los resultados obtenidos acerca de la dependencia de la innovación de procesos, la gestión del conocimiento y la productividad ensancha las investigaciones anteriores y brinda una configuración nueva de como estudiar el fenómeno que viabilice de perfeccionar la exploración futura orientada al problema, pero al mismo tiempo es necesario validarlos en otros ámbitos corporativos y sectores competitivos.

\section{Referencias}

Acuacar (2019). Aguas de Cartagena S.A. E.S.P. "Acuacar". Recuperado de www.acuacar.com

Alarcón, L. \& Campero, M. (1999), Administración de proyectos civiles. Santiago, Chile: Ediciones Universidad Católica de Chile.

Alarcón, L. (1997). Lean construction. Rotterdam, Holanda: Balkema publishers.

Álvarez, K., Serrano, L. \& Bravo, E. (2017). Innovación en Salud: revisión de literatura científica de la última década. Dimensión Empresarial, 15(1), 43-61. DOI: http://dx.doi.org/10.15665/rde.v15i1.559

Arora, A., Cohen, W. \& Walsh, J. (2016). The acquisition and commercialization of invention in American manufacturing: Incidence and impact. Research Policy, 45(6), 1113-1128. DOI: https://doi.org/10.1016/j.respol.2016.02.005

Atal, V. \& Bar, T. (2010). Prior art: To search or not to search. International Journal of Industrial Organization, 28(5), 507-521. DOI: https://doi.org/10.1016/j.jindorg.2009.12.002

Baldwin, C. \& Von-Hippel, E. (2011). Modeling a paradigm shift: From producer innovation to user and open collaborative innovation. Organization Science, 22(6), 1399-1417. DOI: https://www.jstor.org/stable/41303133

Barlow, J., Bayer, S. \& Curry, R. (2006). Implementing complex innovations in fluid multi-stakeholder environments: Experience of Telecare. Technovations, 26(3), 396-406. DOI: https://doi.org/10.1016/j.technovation.2005.06.010

Bettis, R., Helfat, C. \& Shaver, J. (2016). The necessity, logic, and forms of replication. Strategic Management Journal, 3711), 2193-2203. DOI: https://doi.org/10.1002/smj.2580 
Botero-Botero, L. \& Álvarez-Villa, M. (2003). Identificación de Pérdidas en el Proceso Productivo de la Construcción. Revista Universidad EAFIT, 130, 65-78. Recuperado de http://publicaciones.eafit.edu.co/index.php/revista-universidad-eafit/article/view/911

Caro-Paz, R. \& González, D. (2015). Administración de las operaciones. 1a ed. Mar del Plata, Argentina: Ediciones Facultad de Ciencias Económicas y Sociales Universidad Nacional del Mar del Plata.

Chappin, E. \& Ligtvoet, A. (2014). Transition and transformation: A bibliometric analysis of scientific networks researching socio-technical change. Renewable and Sustainable Energy Reviews, 30, 715-723. DOI: https://doi.org/10.1016/j.rser.2013.11.013

Chatterji, A. \& Fabrizio, K. (2014). Using users: When does external knowledge enhance corporate product innovation? Strategic Management Journal, 35(10), 1427-1445. DOI: https://doi.org/10.1002/smj.2168

Coffano, M. \& Tarasconi, G. (2014). CRIOS-Patstat database: Sources, contents and access rules (CRIOS Working Paper No. 1). Milan, Italy: Center for Research on Innovation, Organization and Strategy, Bocconi University. DOI: https://doi.org/10.2139/ssrn. 2404344.

Corsino, M., Mariani, M. \& Torrisi, S. (2019). Firm strategic behavior and the measurement of knowledge flows with patent citations. Strategic Management Journal, 40(7)1-30. DOI: https://doi. org/10.1002/smj.3016.

Cruz, R. \& Koch, S. (2015). Reading and evaluating quantitative research in body psychotherapy. International Body Psychotherapy Journal, 12(2), 154-172. Recuperado de https://www.ibpj.org/archive.php

Dahlander, L. \& Gann, D. (2010). How open is innovation? Research Policy, 39(6), 699-709. DOI: http://dx.doi.org/10.1016/j.respol.2010.01.013

Dalvano, L. \& Hidalgo, A. (2012). Innovation management techniques and development degree of innovations process in service organizations. $R \& D$ Management, 42(1), DOI: https://doi.org/10.1111/j.1467-9310.2011.00663.x 
Damanpour, F., Walker, R. \& Avellaneda, C. (2009). Combinative Effects of Innovation Types and Organizational Performance: A longitudinal Study of service organizations. Journal of Management Studies, 46(6), 650-675. DOI: https://doi.org/10.1111/j.1467-6486.2008.00814.x

Deming, E. (2007). Calidad, competitividad y productividad. Málaga, España: Ediciones Díaz de Santos.

Garzón, M. (2018). La capacidad dinámica de aprendizaje. Desarrollo Gerencial, 10(1), 29-47. DOI: https://doi.org/10.17081/dege.10.1.3009.

Hollon, S. (2006). Randomized clinical trials. En Norcross, J., Beutler, L., \& Levant, R. (Eds.) Evidencebased practices in mental health. Washington, DC: American Psychological Association.

Huh. J, Delorme, D. \& Reid, L. (2006). Perceived third-person effects and consumer attitudes on preventing and banning DTC advertising. Journal of Consumer Affairs, 4O(1), 90-116. Recuperado de https://www.jstor.org/stable/2386056

Jensen, M., Johnson, B., Lorenz, E. \& Lundvall, B. (2007). Forms of knowledge modes of innovation. Research Policy, 36(5), 680-693. DOI: https://doi.org/10.1016/j.respol.2007.01.006

Lampe, R. (2012). Strategic citation. The Review of Economics and Statistics, 94(1), 320-333. DOI: https://doi.org/10.1162/REST_a_00159

Laursen, K. \& Salter, A. (2014). The paradox of openness: Appropriability, external search and collaboration. Research Policy, 43(5), 867-878. DOI: https://doi.org/10.1016/j.respol.2013.10.004

Leiponen, A. \& Helfat, C. (2010). Innovation objectives, knowledge sources, and the benefit of breadth. Strategic Management Journal, 31(2), 224-236. DOI: https://doi.org/10.1002/smj.807

Martin, W. \& Bridgmon, K. (2012). Research methods for the social sciences, Quantitative and statistical research methods-from hypothesis to results. Somerset, $\mathrm{NJ}$ : Wiley. Recuperado de https://n9.cl/8gf4

Mendoza-Betin, J. (2018). Capacidades dinamicas: Un analisis empírico de su naturaleza. Revista MLS Educational Research (MLSER), 2(2), 76-92. Recuperado de https://www.mlsjournals.com/Educational-Research-Journal/article/view/80/185 
Nonaka, I. \& Noburo, K. (1998). The concept of "Ba": Building a foundation for knowledge creation. California Management Review. 40(3), 40-54.DOI: https://doi.org/10.2307\%2F41165942

ISO 9001 (2000). Norma ISO 9001:2000 Sistemas de Gestión de Calidad. Requisitos. Recuperado de https://iso9001calidad.com/iso-9001-2015-sistemas-gestion-calidad-requisitos-21.html

ISO 9001 (2015). Norma ISO 9001:2015. Sistemas de Gestión de Calidad. Requisitos. Recuperado de https://www.iso.org/obp/ui/\#iso:std:iso:9001:ed-5:v1:es

Permana, A., Laksmana A. \& Ellitan, L. (2017). The effect of environmental dynamism, dynamic managerial capabilities, and deliberate organizational learning on the SME performance with dynamic capabilities as mediator variable. International Journal of Advanced Research (IJAR), 5(7), 540-551. DOI: http://dx.doi.org/10.21474/IJAR01/4750

Robayo-Acuña, P. (2016). La innovación como proceso y su gestión en la organización: una aplicación para el sector gráfico colombiano. Suma de negocios, $7(16)$, 125-140. DOI: https://doi.org/10.1016/j.sumneg.2016.02.007

Shu-Mei, T. \& Pei-Shan, L. (2014). The effect of knowledge management capability and dynamic capability on organizational performance. Journal of Enterprise Information Management, 27(2), 158-179. DOI: https://doi.org/10.1108/JEIM-05-2012-0025.

Steensma, H. Chari, M. \& Heidl, R. (2015). The quest for expansive intellectual property rights and the failure to disclose known relevant prior art. Strategic Management Journal, 36(8), 1186-1204. DOI: https://doi.org/10.1002/smj.2279

SIT Innovation. (2019). Método de innovación organizacional. Recuperado de http://es.sitsite.com/metodo/

Vasileiou, K., Barnett, J. \& Young, T. (2013). The Production and use of Evidence in Health Care Service Innovation: A Qualitative Study. Evaluation \& the Health Professions, 36(1), 93-105. DOI: https://doi.org/10.1177/0163278712449622

Wadhwa, A. \& Kotha, S. (2006). Knowledge Creation through External Venturing: Evidence from the Telecommunications Equipment Manufacturing Industry. Academy of Management Journal, 49(4), 819-835. DOI: https://www.jstor.org/stable/20159800 
Wadhwa, A., Bodas I. \& Sarkar, M. (2017). the paradox of openness and value protection strategies: Effect of extramural R\&D on innovative performance. Organization Science, 28(5), 873-893. DOI: https://doi.org/10.1287/orsc.2017.1145

Wensing, M., Bosch, M. \& Grol, R. (2010). Developing and selecting interventions for translating knowledge to action. Canadian Medical Association Journal, 182(2), 85-90. DOI: https://doi.org/10.1503/cmaj.081233 